\title{
Tiotropio mejora la función pulmonar y el control del asma en pacientes con asma leve, moderada y severa
}

\author{
Tiotropium improves pulmonary function and control \\ of asthma in patients with mild, moderate, and \\ severe asthma
}

Pablo Ramón Gil Torres, mD. ${ }^{(1)}$; Carlos Andrés Celis Preciado, MD. ${ }^{(2)}$

${ }^{(1)}$ Residente de tercer año de Medicina Interna, Universidad ICESI, Fundación Valle del Lili. Cali, Colombia.

(2)Internista, Neumólogo, Hospital Universitario San Ignacio, Pontificia Universidad Javeriana. Bogotá, Colombia.

Correspondencia: Pablo Ramón Gil Torres, correo electrónico: pablogil@outlook.com Recibido: 06/07/14. Aceptado: 10/09/14.

\begin{abstract}
Referencia
Rodrigo GJ, Castro-Rodríguez JA. What is the role of tiotropium in asthma?: a systematic review with meta-analysis. Chest. 2015;147(2):388-96.

\section{Pregunta}

En pacientes con asma leve, moderada o severa no controlada a pesar de tratamiento adecuado, ¿cuáles son los efectos de la adición de tiotropio con relación a función pulmonar, control de la enfermedad, riesgo de exacerbaciones y efectos adversos?
\end{abstract}

\section{Diseño}

Revisión sistemática y metaanálisis.

\section{Periodo de búsqueda}

Septiembre de 2014.

\section{Estudios}

Se incluyeron ensayos clínicos controlados (paralelos o cruzados) con al menos cuatro semanas de duración que incorporaron adultos y adolescentes mayores de 12 años con asma estable sintomática, de cualquier grado de severidad, en tratamiento con esteroide inhalado o esteroide inhalado más agonista beta 2 de larga acción, en quienes se empleó tiotropio inhalado $(5 \mathrm{mcg}$ una vez al día por medio del dispositivo Respimat ${ }^{\mathbb{B}}$ o $18 \mathrm{mcg}$ a través del HandiHaler ${ }^{\circledR}$ ), en comparación con cualquier tratamiento.

\section{Desenlaces}

- Primarios: Función pulmonar en términos de $\mathrm{VEF}_{1}$ pico o valle y pico flujo (PEF) en la mañana o en la tarde.

- Secundarios: Control del asma evaluado con el cuestionario ACQ-7 (del inglés Asthma Control Questionnaire), calidad de vida determinada con el cuestionario AQLQ (del inglés Mini-Asthma Quality of Life Questionnaire), uso de medicamento de rescate (disparos/día), días por semana libres de síntomas, exacerbaciones de asma (número de pacientes con uno o más episodios que requirieron 
empleo de esteroides), retiros del estudio (totales y debidos a eventos adversos) y seguridad (eventos adversos y eventos adversos serios).

\section{Métodos}

Se realizaron búsquedas en las bases de datos MEDLINE, EMBASE/Excerpta Medica, CINAHL, Scopus y Cochrane Central Register of Controlled Trials hasta septiembre de 2014, así como las bases de datos de la compañía farmacéutica productora del medicamento.

Ambos autores, de forma independiente, estuvieron involucrados en la selección de los estudios, extracción de la información y evaluación del riesgo de sesgo, la cual se realizó de acuerdo con las recomendaciones del instrumento Cochrane. El análisis fue realizado por intención a tratar.

Los estudios seleccionados fueron agrupados en tres protocolos:

1- Tiotropio como terapia adicional al esteroide inhalado en pacientes con asma leve a moderada, en comparación con esteroide.

2- Tiotropio adicional al esteroide inhalado, en comparación con combinación de esteroide más agonista beta 2 de larga acción inhalados en pacientes con asma moderada.

3- Terapia triple: tiotropio junto a la combinación de esteroide inhalado y agonista beta 2 de larga acción, en comparación con combinación fija en pacientes con asma severa.

La severidad del asma se midió de acuerdo con los criterios GINA (del inglés Global Initiative for Asthma).

\section{Resultados}

En total se incluyeron 13 estudios (duración entre 4 y 52 semanas) con 4.966 pacientes. Seis no fueron publicados. Sólo uno no era patrocinado por la industria farmacéutica. En el instrumento Cochrane todos mostraron un riesgo de sesgo bajo salvo uno, el cual tenía una generación de secuencia poco clara y el cegamiento poseía un alto riesgo de sesgo (tabla 1).

\section{Asma leve}

Diez estudios compararon el tiotropio como terapia adicional al esteroide inhalado, en comparación con esteroide, en pacientes con asma leve a moderada. Los resultados mostraron que la adición de tiotropio mejoró la función pulmonar en términos del PEF en la mañana y en la tarde (cambio promedio de 22 y $24 \mathrm{~mL} / \mathrm{min}$, respectivamente), $\mathrm{VEF}_{1}$ pico y valle (cambio promedio de 150 y 140 $\mathrm{mL}$, respectivamente).

Tiotropio también mejoró los resultados del ACQ-7 y AQLQ, aunque sin llegar a la diferencia mínima clínicamente importante y disminuyó el número de pacientes con al menos un episodio de

Tabla 1. Resultado de los desenlaces primarios evaluados. (tio: tiotropio, ICS: esteroide inhalado, LABA: agonista beta 2 de larga acción).

\begin{tabular}{|c|c|c|c|}
\hline Resultado & $\begin{array}{c}\text { Diferencia promedio entre } \\
\text { tio + ICS vs. ICS (IC 95\%) }\end{array}$ & $\begin{array}{c}\text { Diferencia promedio entre } \\
\text { tio + ICS vs. LABA+ } \\
\text { ICS (IC 95\%) }\end{array}$ & $\begin{array}{c}\text { Diferencia promedio entre } \\
\text { tio + IC + LABA vs. IC + } \\
\text { LABA (IC 95\%) }\end{array}$ \\
\hline $\operatorname{VEF}_{1}$ pico $(\mathrm{mL})$ & $150(110$ a 180$)$ & $20(-10$ a 5$)$ & $120(90$ a 160$)$ \\
\hline $\operatorname{VEF}_{1}$ valle $(\mathrm{mL})$ & $140(110$ a 160$)$ & $20(-20$ a 60$)$ & $80(40$ a 110$)$ \\
\hline PEF mañana (L/min) & $22,0(17,7$ a 26,4$)$ & $6,6(1,6$ a 11,7$)$ & $15,9(7,1$ a 24,8$)$ \\
\hline PEF tarde (L/min) & $24,7(20,4$ a 29,0$)$ & $3,3(-4,8$ a 11,6$)$ & $21,2(11,8$ a 30,5$)$ \\
\hline ACQ-7 (cambio) & $-0,14(-0,19$ a $-0,09)$ & $0,00(-0,07$ a 0,07$)$ & $-0,20(-0,25$ a $-0,09)$ \\
\hline AQLQ (cambio) & $0,07(0,01$ a 0,13$)$ & $-0,12(-0,06$ a 0,18$)$ & $0,12(0,05$ a 0,18$)$ \\
\hline Exacerbaciones (RR) & $0,74(0,57$ a 0,95$)$ & $1,23(0,83$ a 1,83$)$ & $0,70(0,53$ a 0,94$)$ \\
\hline
\end{tabular}


exacerbación del asma (10,5 vs. 13,3\%, respectivamente) con un RR de 0,74 (IC95\% 0,57-0,95) y un NNTB de 36 (IC95\% 19-276).

No se encontraron diferencias significativas en los días libres de síntomas de asma, retiros del estudio y eventos adversos.

\section{Asma moderada}

Cuatro estudios compararon la adición de tiotropio al esteroide inhalado en comparación con el uso de combinación de agonista beta 2 de larga acción con esteroide inhalado en pacientes con asma moderada. Tres de ellos emplearon dosis medias de esteroides y uno dosis altas.

Se observó una mejoría en el PEF en la mañana con el tiotropio aunque de magnitud pequeña $(6,6$ $\mathrm{L} / \mathrm{min}$ ), mientras que en el de la tarde no se evidenció una diferencia significativa, como tampoco la hubo en el $\mathrm{VEF}_{1}$. No se hallaron diferencias en el puntaje ACQ-7, los días libres de síntomas de asma, el número de pacientes con al menos una exacerbación del asma, los retiros del estudio o los eventos adversos.

Los pacientes que recibían la combinación con agonista beta2 de larga acción, experimentaron una reducción significativa en el número de uso de medicamentos de rescate (-0,2 disparos/día) y mejoría en el AQLQ aunque sin llegar a la diferencia mínima clínicamente importante.

\section{Asma severa}

Tres estudios compararon la triple terapia con tiotropio y la combinación de agonista beta 2 de larga acción con esteroide inhalado.

La adición de tiotropio se asoció con una mejoría significativa del $\mathrm{PEF}$ en la mañana $(16 \mathrm{~L} / \mathrm{min})$ $\mathrm{y}$ en la tarde $(20 \mathrm{~L} / \mathrm{min})$ al igual que en el $\mathrm{VEF}_{1}$ pico y valle (120 y $80 \mathrm{~mL}$, respectivamente). Nuevamente, hubo una mejoría en los cuestionarios AQLQ y ACQ-7 sin llegar a la diferencia mínima clínicamente importante.

Además, la triple terapia redujo el número de pacientes que experimentaron al menos una exacerbación del asma (18,2\% vs. $24 \%$, respectivamente), con un RR de 0,70 (IC95\% 0,53-0,94) y un NNTB de 17 (IC95\% 9-99).

No se observaron diferencias en el resto de desenlaces.

\section{Conclusión}

Tiotropio no es inferior al agonista beta 2 de larga acción y es superior al placebo en pacientes con asma moderada a severa que no están controlados adecuadamente por dosis baja a media de esteroide inhalado o dosis altas de esteroide inhalado más agonista beta 2 de larga acción. Los beneficios principales se centran en la función pulmonar, y en los pacientes con asma severa, en el incremento en el control y en la reducción del número de exacerbaciones.

\section{Comentario}

Tiotropio es un anticolinérgico inhalado de larga acción con selectividad y alta afinidad por el subtipo $\mathrm{M}_{3}$ del receptor muscarínico, por lo que su mecanismo de acción es prolongado. Este medicamento inhibe el músculo liso de la vía aérea llevando a broncodilatación prolongada. Además, se han descrito otros efectos antiinflamatorios no broncodilatadores que explican su potencial beneficio (1).

Las señales de transducción intracelular para la regulación muscarínica del tono del músculo liso de la vía aérea, son complejas y existen varios mecanismos moleculares que explican el beneficio de este medicamento solo o combinado con agonistas beta 2 o esteroide inhalado en pacientes asmáticos (2).

En su última actualización de abril de 2015, la guía GINA recomienda el empleo de tiotropio como opción adicional en los pasos 4 y 5 del tratamiento y señala que su principal beneficio radica en la reducción de exacerbaciones (3).

La revisión confirma la recomendación de GINA al señalar que la adición de tiotropio a la combinación fija de esteroide y beta 2 de larga acción en pacientes con asma severa, reduce el número de exacerbaciones, con un NNTB de 17, además de mejorar la función pulmonar. 
Así mismo, Rodrigo y cols. reportan que tiotropio podría tener un papel en los pasos 2 y 3 al mejorar la función pulmonar, con un efecto menos evidente en cuanto al control del asma, la calidad de vida y la reducción de exacerbaciones.

Vale la pena aclarar que los resultados aplican en su mayoría a la dosis de $5 \mathrm{mcg}$ suministrada por el dispositivo Respimat ${ }^{\circledR}$ sin que se hayan encontrado diferencias en los eventos adversos reportados.

De otra parte, al evaluar este estudio es pentinente tener en cuenta varias limitaciones potenciales incluyendo el uso de datos de ensayos que no han sido publicados y evaluados por pares, la dificultad para evaluar la significancia clínica de los cambios así como para lograr la diferencia mínima clínicamente importante, la duración corta de la mayoría de ellos (casi un 40\% duraron menos de 12 semanas) y la mejoría lograda en varios puntos en pacientes que tomaban otros medicamentos a altas dosis (teofilina de liberación sostenida, antileucotrienos, esteroides orales e incluso omalizumab).

Los resultados de tres revisiones recientes de la Colaboración Cochrane permiten precisar un poco más el papel del tiotropio en el manejo del asma (4-6).

La primera de ellas señala que, en comparación con la adición de beta 2 de larga acción, agregar tiotropio al esteroide inhalado no ha mostrado ser superior, por lo que la evidencia acumulada no lo recomendaría (4).
La segunda, que evaluó la adición de tiotropio a esteroide inhalado en comparación con dosis altas de esteroide inhalado, solo encontró un estudio de baja calidad, que por tanto no permite hacer recomendaciones al respecto (5).

Finalmente, la revisión de Anderson y cols. señala que en pacientes que reciben esteroide inhalado, la adición de tiotropio reduce el riesgo de exacerbaciones y mejora la función pulmonar, en comparación con el empleo de la misma dosis de esteroide (6).

\section{Conflictos de intereses}

Los autores declaran no tener conflictos de interés.

\section{Bibliografía}

1. Popescu FD. The pharmacological rationale for the use of inhaled tiotropium in asthma. Pneumologia. 2015;64:52-3.

2. Novelli F, Costa F, Latorre M, Malagrinò L, Celi A, Vagaggini B. Tiotropium: a new therapeutic option in asthma. Monaldi Arch Chest Dis. 2013;79:109-15.

3. From the Global Strategy for Asthma Management and Prevention, Global Initiative for Asthma (GINA) 2015. Disponible en: http://www. ginasthma.org/. Fecha de acceso: 10/09/14.

4. Kew KM, Evans DJ, Allison DE, Boyter AC. Long-acting muscarinic antagonists (LAMA) added to inhaled corticosteroids (ICS) versus addition of long-acting beta2-agonists (LABA) for adults with asthma. Cochrane Database Syst Rev. 2015;6:CD011438.

5. Evans DJ, Kew KM, Anderson DE, Boyter AC. Long-acting muscarinic antagonists (LAMA) added to inhaled corticosteroids (ICS) versus higher dose ICS for adults with asthma. Cochrane Database Syst Rev. 2015;7:CD011437.

6. Anderson DE, Kew KM, Boyter AC. Long-acting muscarinic antagonists (LAMA) added to inhaled corticosteroids (ICS) versus the same dose of ICS alone for adults with asthma. Cochrane Database Syst Rev. 2015;8:CD011397. 